\begin{tabular}{|c|l|}
\hline Title & Femtosecond pump-probe studies of phonons and carriers in bismuth under high pressure \\
\hline Author(s) & Kasami, M.; Ogino, T.; Mishina, T.; Y amamoto, S.; Nakahara, J. \\
\hline Citation & $\begin{array}{l}\text { Journal of Luminescence, 119, 428.432 } \\
\text { https://doi.org/10.1016/.jumin.2006.01.028 }\end{array}$ \\
\hline Issue Date & $2006-07$ \\
\hline Doc URL & http://hdl.handle.net/2115/14424 \\
\hline Type & article (author version) \\
\hline File Information & KasamiPoT u15Revised.pdf \\
\hline
\end{tabular}

Instructions for use 


\title{
Femtosecond pump-probe studies of phonons and carriers in bismuth under high pressure.
}

\author{
M. Kasami, T. Ogino, T. Mishina, S. Yamamoto, and J. Nakahara \\ Division of Physics, Graduate School of Science, Hokkaido University, \\ Sapporo 060-0810, Japan
}

July 30th 2005

\begin{abstract}
We investigate the high pressure phase of $\mathrm{Bi}$ under hydrostatic pressure using pump-probe spectroscopy at pressures up to $3.0 \mathrm{GPa}$, and we observe coherent phonons signal and relaxation signal of photo-excited carriers at $\mathrm{Bi}(\mathrm{II})$ and $\mathrm{Bi}(\mathrm{III})$ phases. The pressure dependence of the coherent phonons shows that the amplitude of coherent phonons is extremely small and the frequency of coherent phonons changes at high pressure phases. As results from our experiment, we obtain its frequencies are $2.5 \mathrm{THz}$ and $2.2 \mathrm{THz}$ at $\mathrm{Bi}$ (II) and $\mathrm{Bi}(\mathrm{III})$, respectively. Furthermore, photo-excited carrier relaxation indicates drastic changes near 2.5 GPa. Bismuth transforms from semimetal to semiconductor near 2.5 GPa, and band-overlapping between at L-point and at T-point disappears. We consider that the drastic changes of the photo-excited carrier relaxation are strongly correlated with the band-overlapping disappearing.
\end{abstract}

Keywords:

Coherent phonon, photo-excited carrier, bismuth, high pressure, band-overlapping. Masashi Kasami,

Division of Physics, Hokkaido University,

Sapporo 060-0810, Japan

FAX: 81(Japan)-11-706-4926

E-mail: kasami@phys.sci.hokudai.ac.jp 


\section{Introduction}

Bismuth is one of the most studied elements for unique electrical transport properties and applications. Various high pressure researches in Bi have been reported. Pressure effects on the electrical properties have been investigated with magnetoresistantce, Hall effect, and electrical conductivity $[1,2,3]$. Phase diagrams have been determined using differential thermal analysis by Klement et al. [4]. Crystal structures at high pressure phases have been measured using X-ray diffraction [5], but the structure in Bi(III) phase has been unknown for the last few decades. Mcmahon and co-workers has revealed that $\mathrm{Bi}(\mathrm{III})$ phase takes unique "guest-host" structure in 2000 [6].

The developments of laser technology enable us to generate and detect real-time lattice vibration and relaxation of photo-excited carriers. Coherently generated lattice vibration by pump-probe spectroscopy is called coherent phonons and they are observed in various semimetals and semiconductors. The coherent phonons and the photo-excited carriers relaxation in $\mathrm{Bi}$ have been reported in low temperature and high excitation regime $[7,8]$. However there is no report on those phenomena in high pressure region. The combination of high pressure and pump-probe spectroscopy constitutes a powerful method to investigate real-time variation of phonons and carrier dynamics superior to Raman spectroscopy in low frequency region.

In this report, we perform pump-probe spectroscopy in $\mathrm{Bi}$ single crystal under hydrostatic pressure up to $3.0 \mathrm{GPa}$, and we investigate the coherent phonons and the photo-exited carriers relaxation at high pressures. We observe that the amplitude of coherent phonons extremely decrease, and its frequency changes at high pressure phases. We determine that the frequencies of coherent phonons are $2.5 \mathrm{THz}$ and $2.2 \mathrm{THz}$ at $\mathrm{Bi}(\mathrm{II})$ and at Bi(III) high pressure phases. Furthermore, the photo-excited carrier relaxation changes drastically near 2.5 GPa.

\section{Experiment}

A single crystal of $\mathrm{Bi}$ with lateral dimensions of $200 \mu \mathrm{m}$ and thickness of $100 \mu \mathrm{m}$ is 
prepared. Cleaved surface perpendicular to trigonal face is used to examine pump-probe spectroscopy. The laser pulses are generated by a mode-locked Ti:sapphire laser. Pulse width, center wavelength and repetition rate are about $100 \mathrm{fs}, 80 \mathrm{MHz}$ and $\lambda=790 \mathrm{~nm}$ $(\mathrm{h} v=1.57 \mathrm{eV})$, respectively. A standard reflection-type of time resolved pump-probe technique is employed. Pump and probe beams are polarized orthogonal to each other to avoid the scattered pump beam, and diameter of laser spot is about $10 \mu \mathrm{m}$ on the samples. The excitation power density is about $50 \mu \mathrm{J} / \mathrm{cm}^{2}$. The reflectivity change $\Delta R / R$ is measured as a function of the delay time by scanning the optical path length of the probe beam.

A diamond anvil cell (DAC) is used as a pressure generation source and pressure is determined by the ruby fluorescence method. To examine a small sample laded in the DAC, we use microscope pump-probe system in this experiment. The schematic diagram of microscope pump-probe system is shown in Fig. 1. Pump and probe beam are reflected by dielectric mirror and focused on the sample in the DAC. Laser spot and the inside of the DAC are monitored by the CCD camera while measuring.

Figure 2(a) shows schematic diagram of optical setup near sample position. A mixture of methanol and ethanol 4:1 is used as a pressure transmitting medium. To confirm the heating effects by laser beam on the sample, thermocouple is equipped to diamond. The sample is fairly good thermal contact with the diamond, however, we cannot observe the significant heating effect. Figure 2(b) shows pump and probe signals of the single crystal of $\mathrm{Bi}$ measured in a DAC (under $1 \mathrm{kbar}$ ) and in an atmosphere. In the DAC, laser pulses pass through the diamond and the pressure-transmitting medium, however we cannot observe significant differences both signals in the DAC and in an atmosphere.

\section{Experimental Results}

Figure 3 shows the pump and probe signals in the single crystal of $\mathrm{Bi}$ under hydrostatic pressure ranging from atmospheric pressure to 3.0 GPa. The horizontal dotted lines indicate baselines of each signal and the spikes at 0 ps are caused by the transient grating effect. The 
damped oscillatory component of pump and probe signal represents coherent $\mathrm{A}_{1 \mathrm{~g}}$ phonon mode and non-oscillatory background component shows relaxation of photo-excited carriers. The coherent phonons signal is clearly observed at atmospheric pressure and 1.0 GPa. At atmospheric pressure and 1.0 GPa, the relaxation signal of photo-excited carriers rises at $0 \mathrm{ps}$, and it is decaying rapidly with the time constant on the order of $10 \mathrm{ps}$. Increasing the pressure, the pump and probe signals indicate drastic changes at $2.6 \mathrm{GPa}$ and 3.0 GPa. The amplitude of coherent phonons is extremely small, and the photo-excited carrier relaxation shows drastic changes. The relaxation signal of photo-excited carriers falls to the lowest point and it indicates sufficiently slow relaxation. There are no significant changes of photo-excited carriers relaxation between at 2.6 GPa and at 3.0 GPa, therefore it is considered that the drastic change of the relaxation is not caused by structural phase transition from $\mathrm{Bi}(\mathrm{II})$ to $\mathrm{Bi}(\mathrm{III})$.

To investigate the pressure dependence of the coherent phonons, we use time modulation technique to highlight the coherent phonons component of pump and probe signal. The time derivative of pump and probe signals at various pressures are shown in Fig. 4(a). The amplitudes of coherent phonons at 2.7 GPa and 2.8 GPa decrease to about one tenth of the signal at atmospheric pressure. The Fourier transform (FT) spectra of time domain signals are displayed in Fig. 4(b). The peak frequency of the FT spectrum is $2.9 \mathrm{THz}$ at atmospheric pressure, and it agrees well with the $\mathrm{A}_{\lg }$ phonon frequency measured by the Raman spectroscopy [9] and time domain data [10]. As the pressure increases, the frequency of coherent phonons changes from $2.9 \mathrm{THz}$ to $2.5 \mathrm{THz}$ at $2.7 \mathrm{GPa}$. Then, the secondly frequency change is observed at $2.8 \mathrm{GPa}$, and the peak frequency is about $2.2 \mathrm{THz}$ at this pressure. Bi transforms from rhombohedral to monoclinic $\mathrm{Bi}(\mathrm{II})$ at $2.5 \mathrm{GPa}$ (at room temperature). $\mathrm{Bi}(\mathrm{III})$ phase appears at $2.8 \mathrm{GPa}$, and its structure takes unique guest-host structure [6]. Coherent phonons are affected by the change of the crystal structures, therefore, we consider that the frequency shifts are caused by the lattice potential change at structure phase transition. It is expected to occur phonon lifetime change, but we cannot observe the significant change of it. 


\section{Discussions}

Bismuth is a semimetal at atmospheric pressure (at room temperature), and band-overlapping exists between valence band at T-point and conduction band at L-point. It is known that $\mathrm{Bi}$ transforms from semimetal to indirect semiconductor near $2.5 \mathrm{GPa}$, and consequently, the band-overlapping disappears. Schoenlein et al. have investigated wavelength dependence of pump and probe signals in GaAs ranging from 1.55 to $2.14 \mathrm{eV}$, and their results show that photo-excited carrier relaxation extremely changes when the photon energy of a laser pulse coincide with any electronic band [11]. We investigate the wavelength dependence of pump and probe signals in Bi ranging from $1.51 \mathrm{eV}$ to $1.70 \mathrm{eV}$ at atmospheric pressure, and we cannot confirm the significant changes of photo-excited carrier relaxation. Since the pressure dependence of phonon frequency show slight change, it is considered that the electronic band structures are almost unchanged by the structural phase transition. The drastic changes of photo-excited carrier relaxation occur when band-overlapping disappears at semimetal-semiconductor transition. Therefore, we consider that the drastic changes of photo-excited carrier relaxation above $2.5 \mathrm{GPa}$ are correlated with the band-overlapping disappearing. Further theoretical and experimental studies are needed to clear our experimental results.

\section{Conclusions}

We perform pump-probe spectroscopy in Bi under hydrostatic pressure. We observe coherent phonons and relaxation of photo-excited carriers in high pressure phases of $\mathrm{Bi}$ (II) and $\mathrm{Bi}(\mathrm{III})$. We observe the amplitude of coherent phonons is extremely small and the phonon frequency changes at high pressure phases. We determine that frequencies of coherent phonons are $2.5 \mathrm{THz}$ and $2.2 \mathrm{THz}$ at $\mathrm{Bi}(\mathrm{II})$ and $\mathrm{Bi}(\mathrm{III})$, respectively. Coherent phonons are sensitive to the structure changes, therefore, we consider that the frequency shifts are caused by the lattice potential change at structural phase transition. Moreover, drastic changes of the photo-excited carrier relaxation are observed near 2.5 GPa. Bismuth 
transforms from semimetal to semiconductor near $2.5 \mathrm{GPa}$. The drastic change of photo-excited carrier relaxation is correlated with the disappearing of the band-overlapping at semimetal-semiconductor transition.

\section{Acknowledgments}

The authors thank Mr. T. Kuwajima and Mr. H. Nomura of Technical Laboratory of Lamina, Graduate School of Science, Hokkaido University for sample preparation. The experimental assistance of Mr. Y. Ishibashi is gratefully acknowledged. The authors acknowledge Osaka Asahi Metal MFG. co., ltd for supplying the 6N bismuth sample. This work was partially supported by Hokkaido University, The 21st Century COE Program “Topological Science and Technology”. 


\section{REFERENCES}

[1] J. R. Vaisnys and R. S. Kirk, J. Appl. Phys. 38, 4335 (1967).

[2] A. A. Averkin, Yu. G. Vorov, G. A. Ivanov, and A. R. Regel, Sov. Phys. Solid State 13, 309 (1971).

[3] M. Lu, R. J. Zieve, A. V. Hulst, H. M. Jaeger, T. F. Rosenbaum, and S. Radelaar, Phys. Rev. B 53, 1609 (1996).

[4] W. Klement, A. Jayaraman, and G. C. Kennedy, Phys. Rev. 131, 632 (1963).

[5] J. H. Chen, H. Iwasaki, and T. Kikegawa, High Pressure Research 15, 143 (1996).

[6] M. I. McMahon, O. Degtyareva, and R. J. Nelmes, Phsy. Rev. Lett. 85, 4896 (2000).

[7] M. Hase, K. Miozoguchi, H. Harima, S. Nakashima, and K. Sakai, Phys. Rev. B 58, 5448 (1998).

[8] O. V. Misochko, M. Hase, K. Ishioka, and M. Kitajima, Phys. Rev. Lett. 92, 197401-1 (2004).

[9] J. S. Lannin, J. M. Calleja, and M. Cardona, Phys. Rev. B 12, 585 (1975).

[10] T. K. Cheng, J. Vidal, H. J. Zeiger, G. Dresselhaus, M. S. Dresselhaus, and E. P. Ippen, Appl. Phys. Lett. 59, 1923 (1991).

[11] R. W. Schoenlein, W. Z. Lin, E. P. Ippen, and J. G. Fujimoto, Appl. Phys. Lett. 51, 1442 (1987). 


\section{FIGURES}

Fig. 1. A schematic diagram of micro pump-probe system. Pump and probe beams are reflected by the dielectric mirror and focused on the sample in diamond anvil cell (DAC). Inside of the DAC is monitored by CCD camera while measuring.

Fig. 2. (a) Schematic diagram of optical setup and sample. Single crystal of Bi is loaded in a DAC with Ruby chips. (b) Pump and probe signals of Bi measured in a DAC (under 1 kbar) and in an atmosphere. We cannot observe significant difference on signal in the DAC for comparison with the signal in an atmosphere.

Fig. 3. The pressure dependence of pump-probe signal. The pump-probe signal of $\mathrm{Bi}$ is measured at various pressures ranging from atmospheric pressure to $3.0 \mathrm{GPa}$. The horizontal dotted lines indicate baselines of each signal. We observe damped oscillation component of coherent phonons and non-oscillation background component of photo-excited carriers relaxation are clearly observed at atmospheric pressure and at 1.0 GPa. However, the amplitude of coherent phonons is extremely small and the photo-excited carriers relaxation is decaying to the negative direction at $2.6 \mathrm{GPa}$ and $3.0 \mathrm{GPa}$.

Fig. 4. (a) The pressure dependence of coherent phonons. Time derivatives of pump-probe signals at atmospheric pressure, at $2.7 \mathrm{GPa}$ and at $2.8 \mathrm{GPa}$ using time modulation technique to highlight coherent phonons. (b) Fourier transform spectra in each signal. Frequency of coherent phonons at atmospheric pressure is $2.9 \mathrm{THz}$, however, frequency changes to about $2.5 \mathrm{THz}$ at $2.7 \mathrm{GPa}$ in $\mathrm{Bi}(\mathrm{II})$ phase. Further frequency change is occurred at $2.8 \mathrm{GPa}$ in $\mathrm{Bi}(\mathrm{III})$ phase and the frequency is about $2.2 \mathrm{THz}$. 
Fig.1

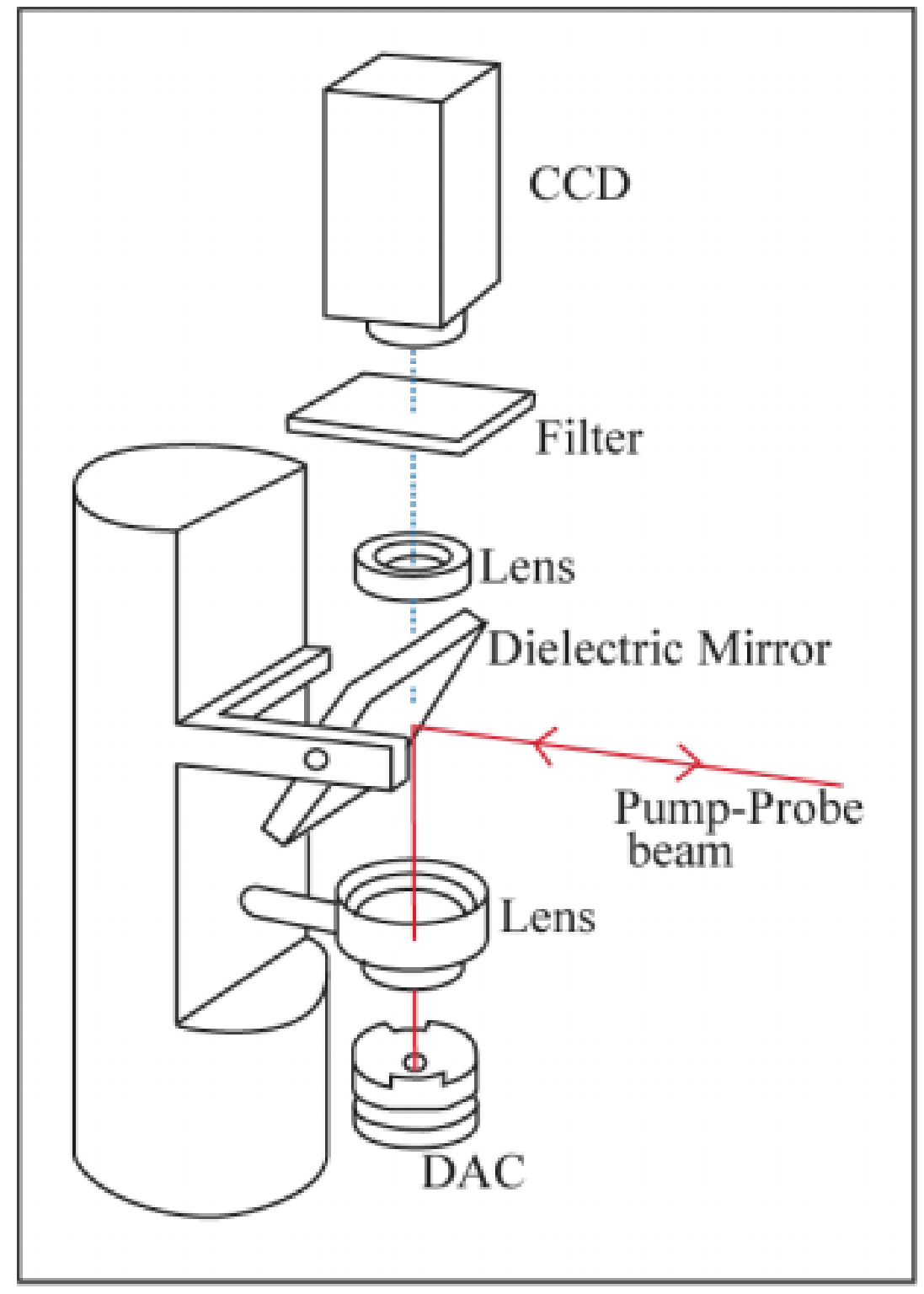


Fig. 2
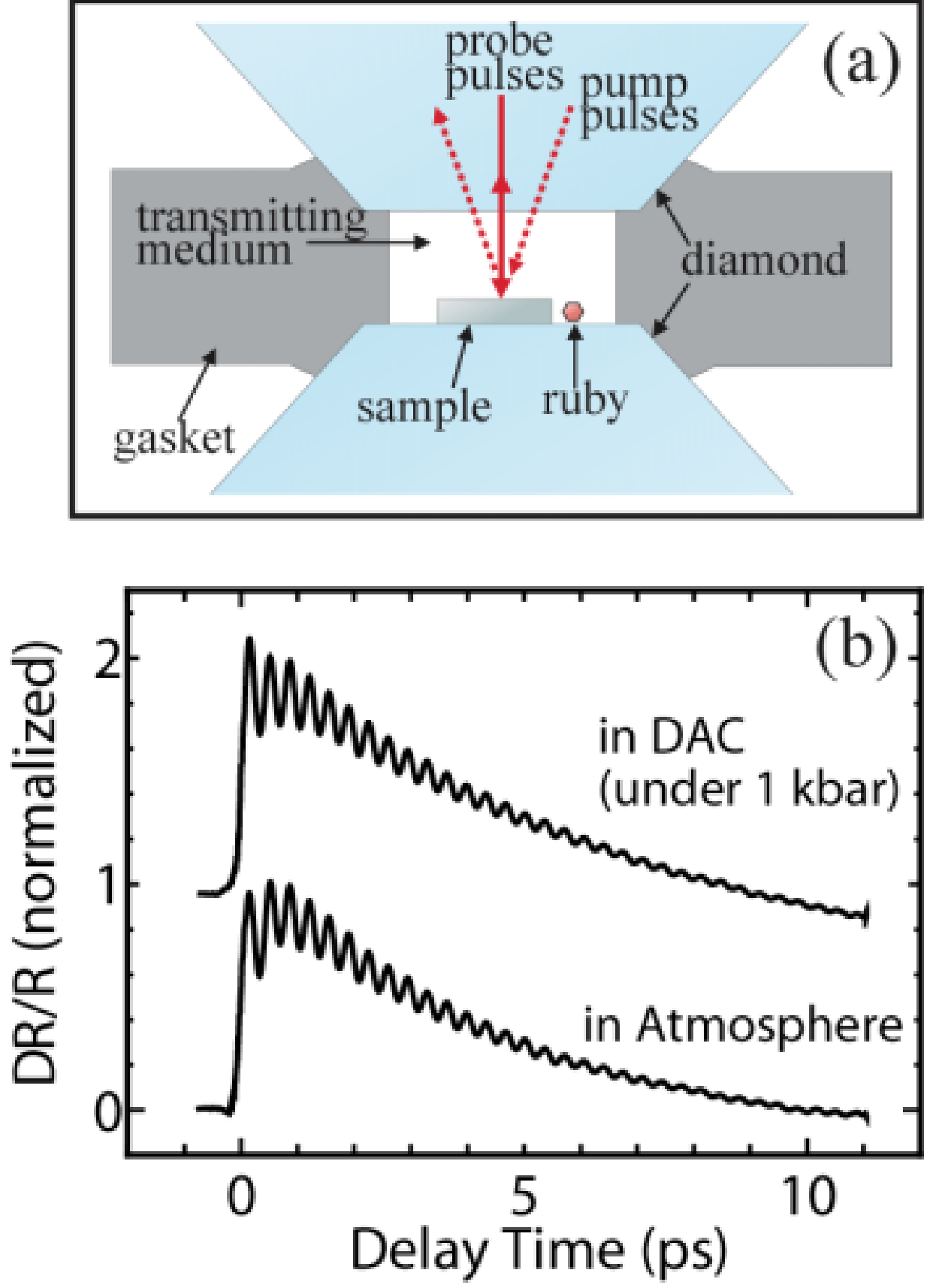
Fig. 3

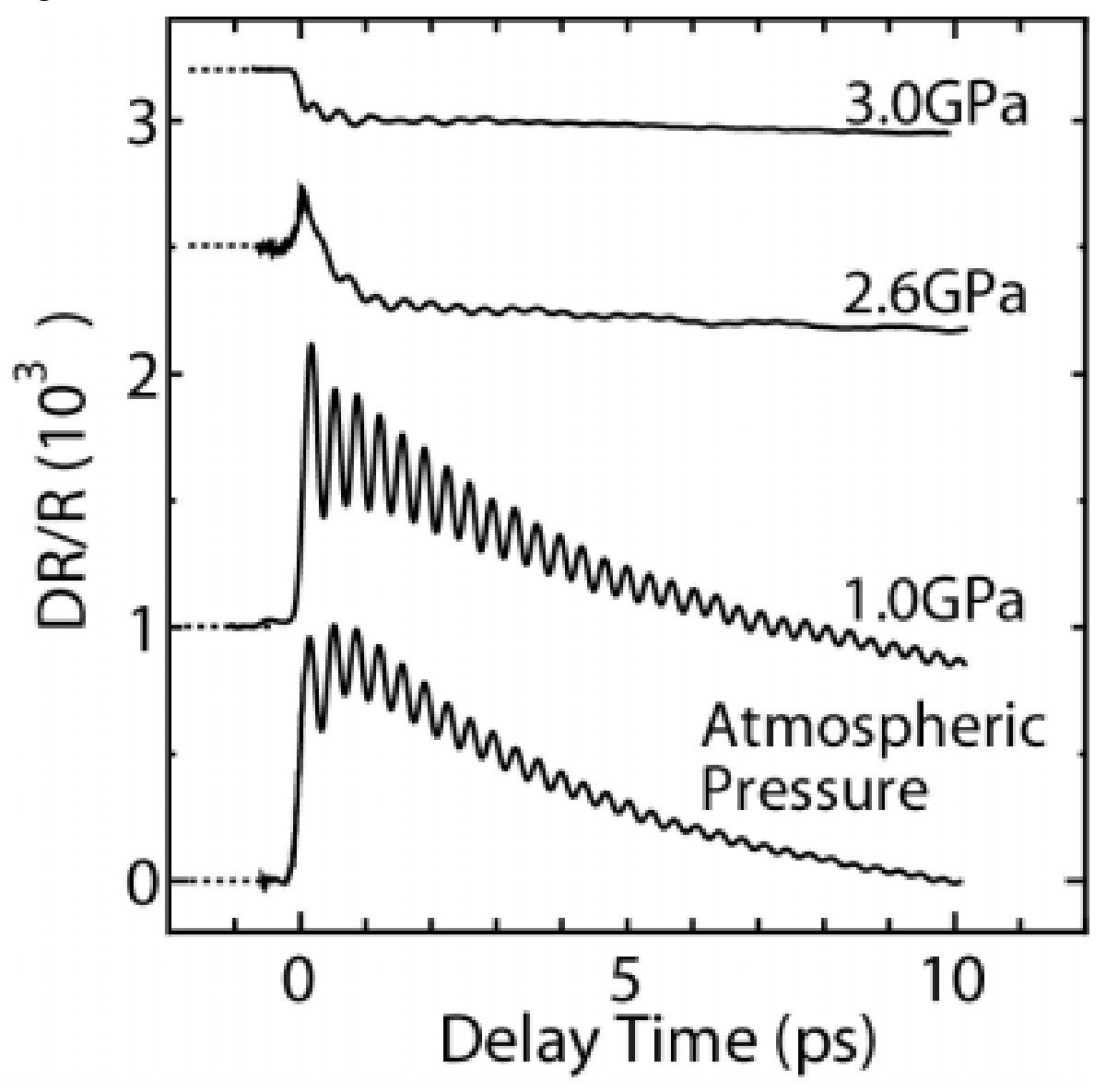


Fig. 4
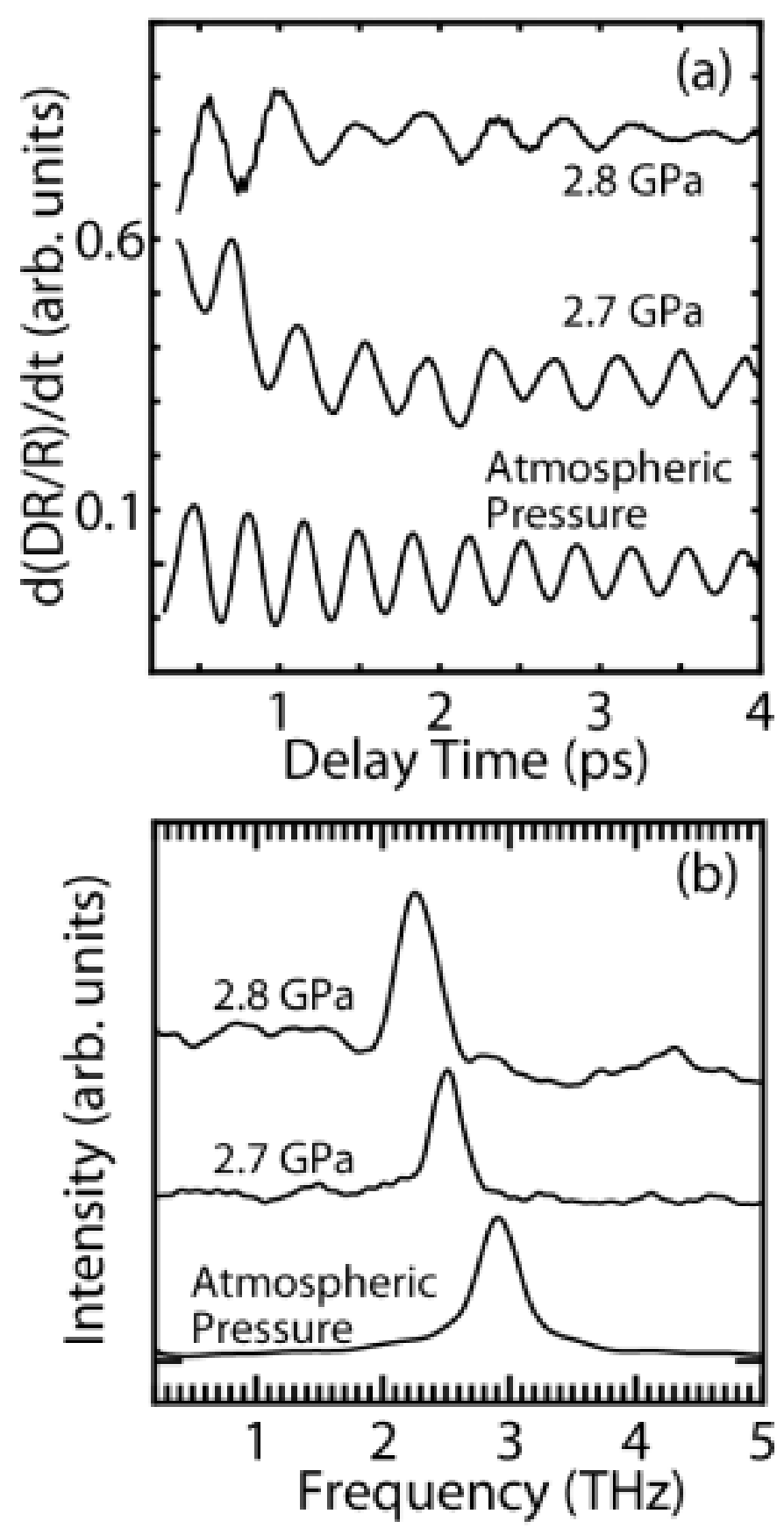